\title{
In-Game Instructions: The Extent of Their Usefulness in Enhancing the Vocabulary Acquisition of ESL Learners
}

\author{
https://doi.org/10.3991/ijet.v15i04.11647 \\ Tan Pei Qin \\ SJK(C) Maran, Pahang, Malaysia \\ Tan Kim Hua ${ }^{(凶)}$ \\ Universiti Kebangsaan Malaysia, Bangi, Malaysia \\ kimmy@ukm. edu.my
}

\begin{abstract}
The contributions of video games to learning, including vocabulary acquisition, have been acknowledged over the past few decades. Ingame instructions may account for the majority of such contributions. This study checks whether such instructions can pique the curiosity of participants and subsequently enhance their vocabulary acquisition. Ten 11 to 12-year-old pupils were recruited for a month-long video game experiment. Three instruments, including vocabulary tests, interview questions and observation checklists, were employed. The qualitative data were analysed to determine the key aspects of in-game instructions and how they enhance the vocabulary acquisition of these pupils. Findings show that not all participants have gained additional vocabulary from in-game instructions. Whilst all participants were interested in playing the video game, only four of them showed curiosity towards the in-game instructions. Therefore, the usefulness of in-game instructions fundamentally depends on the attitudes of players towards the game, the suitability of the language level being used in the game and the players' demand for such instructions.
\end{abstract}

Keywords-In-game instructions, vocabulary acquisition, curiosity

\section{Introduction}

English is the second language in many countries, including Malaysia. To master a non-native language, suitable skills and techniques must be applied. Although video games can help people learn a non-native language, they have been criticised in terms of their usefulness and effectiveness. Therefore, how players react to video games and how such games enhance these players' vocabulary acquisition need to be examined.

With millions of players worldwide, video games have become part of the global culture [17]. Before the digital revolution, people completed their education via chalkand-talk lessons, whereas Generation $\mathrm{Z}$ people, which cover those people between aged 13 years and 17 years who have never experienced a world without video games 
[8], [17], are given unlimited Internet access and use digital devices to attend online classes and engage in online entertainment activities.

However, those people who spend their time playing video games outnumber those who spend their time studying online. Attractive game design, advertising, availability at one's fingertips and social connectivity are some factors that contribute to the growth of the video game culture. With the rapid progress of the global gaming culture, researchers have been constantly looking for ways to utilise such culture to benefit the education of young generations even though an extended exposure to video games has been proven to lead to Internet gaming disorders [1].

Video games, which are irresistible to people of all ages, have been proven to contribute to learning in various domains [22]. For instance, these games create a positive environment for learning a new language by lowering the anxiety of players, increasing their motivation and encouraging expression [40], [20]. Some researchers reveal that communication in video games, such as through text and voice chats, can help players acquire new vocabulary and improve their social and cognitive skills [17]. Gaming can also foster critical thinking as revealed in a survey of gamer and non-gamer groups, with the former group demonstrating better critical thinking skills compared with the latter group [16].

Previous studies have attempted to identify the effectiveness of video games, which characteristics or features can be incorporated into classroom teaching. Such integration is crucial in adapting the current education system to a world of advanced technology and to promote the academic efforts of the young generation.

\subsection{Issues}

ESL learners consider vocabulary as the most fundamental part of their learning given that a strong vocabulary can foster one's understanding, speaking and reading abilities. However, the traditional way of teaching vocabulary by 'spoon feeding' has been proven to be largely ineffective for children nowadays given their short concentration spans. Moreover, many teachers are not very confident with the current best practices in vocabulary teaching [19]. Therefore, a new technique that can engage children in learning and mastering vocabulary must be devised.

With the rapid development of the gaming culture across the globe, researchers have attempted to establish a relationship between playing video games and mastering vocabulary. Between educational and non-educational games, the latter promotes incidental learning, which is important for vocabulary acquisition given that most words are acquired in incidental settings. Many researchers have explored the potential vocabulary acquisition environment in video games, especially in massively multiplayer online games (MMOGs) [18] or massive multiplayer online role-playing games (MMORPGs) [40], whereas others have focused on the designs of games that require players to interact with one another. Out-of-game texts are vital in helping players develop strategies, complete quests and search for certain items in games. These texts can be easily seen by gamers by searching websites or communicating with game experts. Therefore, by exposing gamers to new vocabulary whilst playing, video games can promote vocabulary acquisition. 
Aside from out-of-game texts, the use of in-game instructions in promoting the academic development of players also warrants further exploration. To the best of the researcher's knowledge, this study is the first to explore the efficiency of in-game instructions given to players by focusing on how these instructions are presented. This work aims to examine the authenticity of the claim that video game instructions can help gamers indirectly acquire new vocabulary. This research also aims to investigate whether one's motivation to learn new words can be increased by challenging and motivating video game instructions.

\subsection{Aim of research}

The objectives of this study are:

- To identify whether in-game instructions can arouse the curiosity of gamers to learn the words they encounter during their gameplay

- To investigate whether in-game instructions can familiarise participants with unknown words

To achieve these objectives, this study seeks to answer the following questions:

- How do the research participants react to in-game instructions containing difficult words that they encounter during their gameplay?

- What are the vocabulary test results of these participants before and after playing the video game for a month?

\section{Literature Review}

\subsection{Vocabulary in video games}

The success of vocabulary acquisition through video games depends on the ways how words are presented in these games. Learners can acquire many types of vocabulary by playing video games. Some participants in this study commented that they were proud of the specialised vocabulary they learned through video games, thereby indicating that learners can gain different types of vocabulary from various types of games.

Given that players may have different learning preferences, the elements of a game must be well-designed to achieve an effective vocabulary acquisition. Serious games, such as online and offline games contain many difficult words, whilst their designs require players to focus in order to move on to another level [2]. In this way, these games are both entertaining and instructive. Drill- and task-type games provide players with sufficient exposure to words through multiple texts and focus on the meanings of words rather than on the words themselves [25].

According to Plass and Kaplan [12], the vocabulary presented in games should focus on the cognition of players. A good game provides players with opportunities to learn in meaningful and relevant contexts where important information is being 
shared on time and fit well with the needs of gamers. The knowledge received by players from these games can also be transferred to a school context in several ways. Firstly, scaffolding, which should be given at the right time, must fall within the learners' zone of proximal development to help them solve in-game tasks. Secondly, a dynamic assessment and feedback process must be performed based on the learning goals of an individual. Thirdly, the information design should consider semiotics or choice of signs as learning content. Fourthly, the learning mechanics should be closely aligned with the learners' learning goals. The mapping of gestures and movements is an interesting key feature of gaming. Gee [21], [15] proposed three key principles that need to be observed in game design, namely, 'just in time', 'on demand' and 'situated meanings'. These principles strongly support the findings of the above studies on the impact of gaming principles on vocabulary acquisition.

Good games engage players not only by letting them play the game but also by driving their interest to develop their cognitive skills through reading and understanding texts. Games usually have two types of texts, namely, in-game and game-related texts. A corpus analysis reveals a high degree of lexical sophistication, lexical diversity and syntactic complexity in in-game texts from World of Warcraft [23]. However, in-game texts have been associated with images, actions, goals and dialogues instead of definitions [14]. Game-related texts, also known as paratexts, include video tutorials, walkthrough, fan fiction and fan art that are found in online gaming communities and used as practical tools for strategy training. These highly sophisticated texts cover multiple genres, contain a rich amount of lexical items and show syntactic structures [40]. Therefore, language literacy can be improved by reading paratexts [13].

Another study revealed that offline games limit the interaction of gamers with ingame texts, but observing the playing behaviour of other gamers may help them acquire new vocabulary and expose themselves further to a new language. However, the high level of cognitive load induced by the interactivity of the game can hinder one's recall of vocabulary [11]. Single-player games allow gamers to freely observe the playing behaviour of others and free themselves from peer pressure. This theory echoes the social aspects of agency, which Bandura [3] categorised into personal agency (by individual) and proxy agency (influenced by others).

Thorne et al. [38] identified quest texts as important sources of in-game information. These texts provide instructions that help players carry out specific ingame tasks. Therefore, the readability and complexity of these texts must be suitable to players of different ages. A study of the semiotic ecology and linguistic complexity of games revealed that the D-Level scale can help identify the level of quest texts. For example, the highest level (level 7) indicates that a quest text consists of more than one structure from levels 1 to 6 . In this way, it is appropriate for children between the ages of $12-14$ as the lexical items are potentially difficult for this age group to understand.

Some researchers argued that vocabulary learning may be influenced by game mechanics because when learners are confused with these mechanics, they will lose their focus on language learning [4]. The usefulness of the vocabulary being used 
throughout the game may also differ depending on the types of games and the learners themselves.

\section{2 'Criminal case' game}

Criminal Case, a detective-themed hidden object game produced by Pretty Simple in 2012, was used in this work to manipulate the effect of in-game instructions on the vocabulary acquisition of primary ESL learners. This game can be easily controlled by participants with minimal computer skills just by simple clicking. Criminal Case was selected for this study for three reasons. Firstly, the language and vocabulary used in the in-game instructions were both challenging and motivating for the target group. Secondly, Criminal Cases tests the problem-solving skills of players by requiring them to collect evidence and participate in case analyses. Thirdly, similar to other puzzle video games, Criminal Case uses 'lives' to limit the number of allowable attempts of players and requires them to obtain a high score or satisfy certain criteria before moving on to the next level. Moreover, a short time used in searching all things mentioned will be awarded a diamond. A diamond can be used to unlock the game to go to the next level. When searching for hidden objects in a crime scene, players may not be able to understand the difficult words presented in the game, such as the names of certain items. In other words, this game provides players with some hints in exchange for their time and points.

Criminal Case involves searching for hidden objects, finding evidence, solving puzzles and participating in case analyses. This game also requires players to read plenty of text, especially when analysing cases along with inspectors and the police. Players can pause the game to read the case. The search for potential evidence, such as a pocket watch or a domino (Figure 1), also motivates players to follow the ingame instructions. Technical instructions, such as 'Click on the finish button to get the result', 'Select your game partner', 'Click anywhere to begin the investigation' and 'Solve the puzzle (Figure 2)' and other gameplay instructions are also used. As described below, the gameplay instructions in Criminal Case are presented in three forms, namely, in dialogues, short sentences and words or phrases. Most of the ingame dialogues are presented as narratives (Figure 3) to help the players choose which actions to take to complete a case. A discussion among experts reveals that such narration provides players with new words, such as 'involved' and 'register'. This is useful in guiding players to understand better the case analysis. The motivation to identify the real murderer will urge them to read those instructions. An example of a short sentence used in the game to direct the player is 'Talk to Joe Stern (Figure 4)'. 


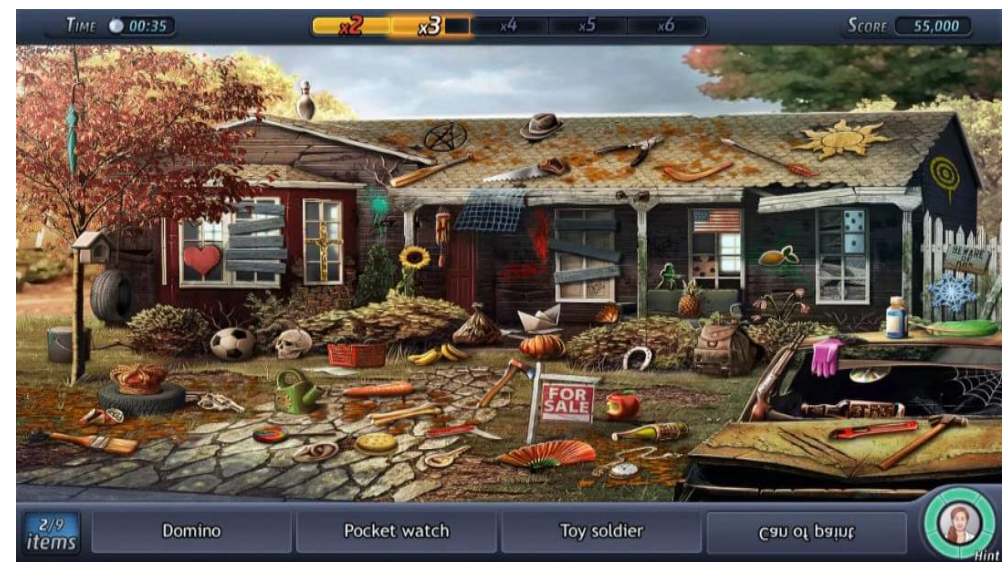

Fig. 1. Instructions in words and phrases

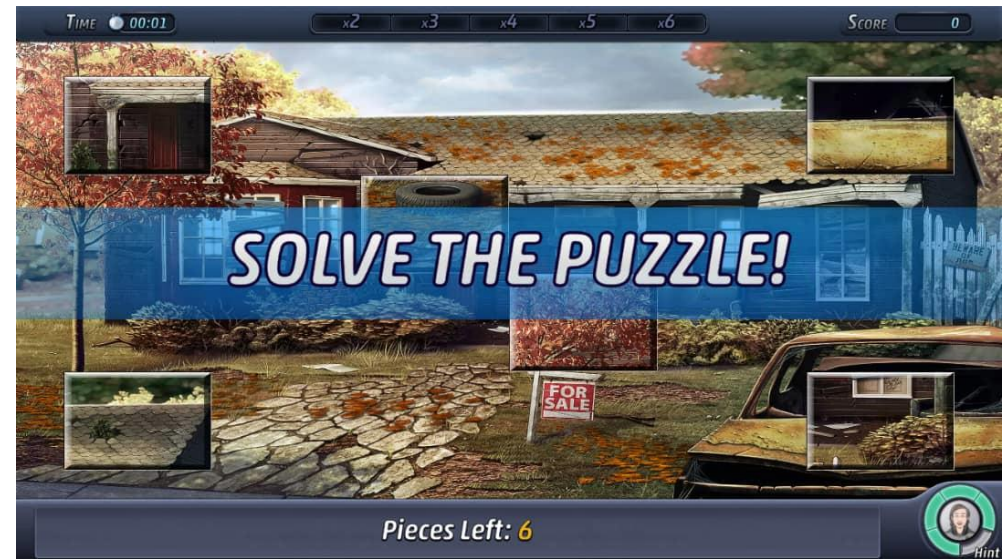

Fig. 2. Technical Instructions in short sentences

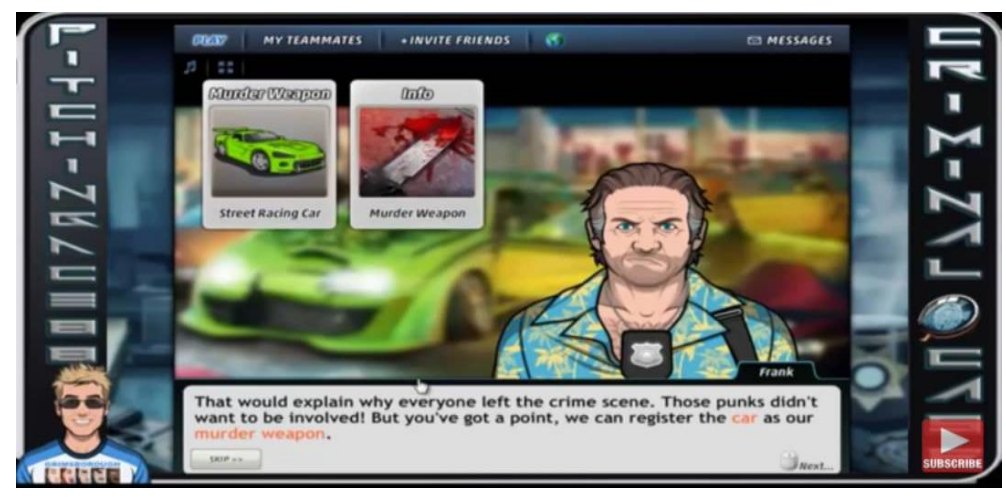

Fig. 3. Narrative instructions in long sentences 


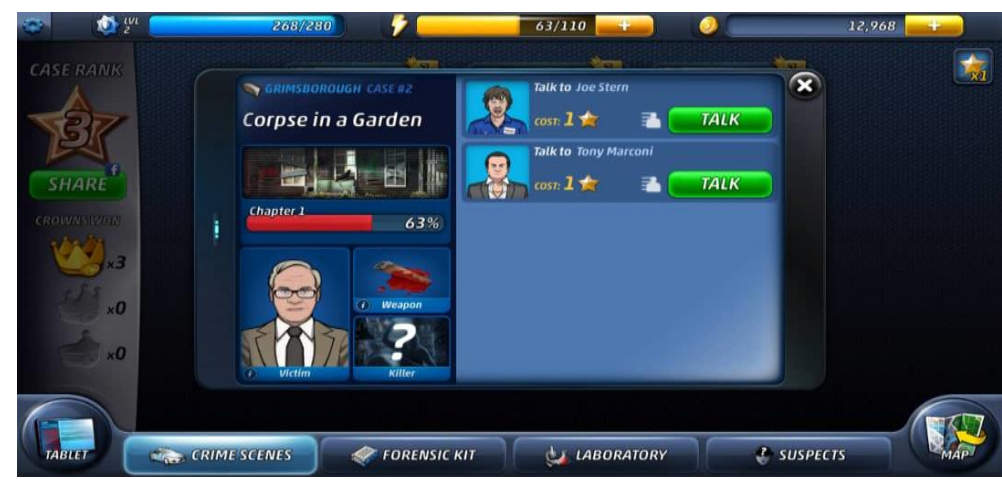

Fig. 4. Gameplay instructions in short imperative sentences

\subsection{Significance of the study}

Based on previous studies, most of the research findings revealed that video games can benefit vocabulary acquisition in various ways and significantly influence the English language skills of players. However, there is no study conducted to identify the impacts of in-game instructions on vocabulary acquisition. While sound effects, attractive graphics, interaction features and many other game designs had been proven to be effective in enhancing vocabulary learnt, instructions in game were not the focus of researchers in the field. This opens up a new dimension for researchers and educators to explore. It provides a new insight into effective teaching and learning approaches in the twenty first century.

\section{$3 \quad$ Methodology}

\subsection{Research design}

A design that minimises bias and experimental errors is considered a good design [9]. The exploratory research design was employed in this study, and a few research instruments, including observation checklists, face-to-face interviews and vocabulary tests were employed. Observation checklists were used to measure the complexity of the social phenomena by recording the behaviour of the participants. These instruments also allowed researchers to be inductive and open to discovery. Meanwhile, face-to-face interviews are useful to obtain the honest opinions and perceptions of the research participants, allow researchers to ask probing questions and prevent the responses of each participant from being influenced by the opinions of others. Researchers can also obtain quality responses from their interviewees by observing and interpreting their facial expressions and body language [4]. A good vocabulary test can successfully assess and evaluate the number of words learnt by the participants. 


\subsection{Theoretical framework}

Garries et al [35] introduced a generic model about the input-process-outcome framework of game (Figure 5). The key components of this framework is the game cycle which it highlights the iterative process of user judgement, user behaviour and system feedback. The inputs include instructional content and game characteristics whilst outcome is the learning outcome.

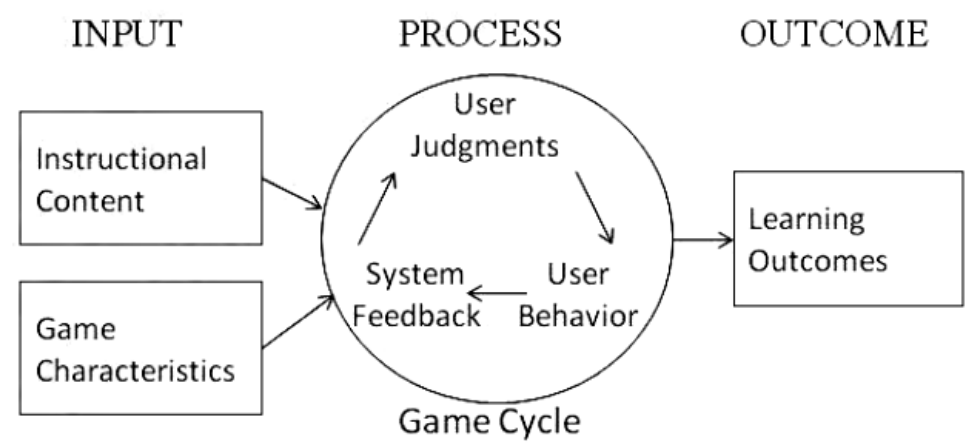

Fig. 5. Input-process-outcome framework (Garris, 2002)

This model can explain the learning process happens in serious game and it fits well into modern didactic theories [24]. It highly emphasises motivation of the learner in playing the game which it is in line with this current study. Nevertheless, in-game instruction is chosen to be the research focus so the input of game characteristics is weigh lesser. Therefore, this model is suitable to be adapted and integrated to support the purpose of this research study.

\subsection{Conceptual framework}

The conceptual framework of this research emphasises the possibility for the participants to improve their vocabulary acquisition by reading in-game instructions in video games. Learning theories and the vast studies on the potential of different features of video games towards learning support this rationalisation. Though emphasis in past studies are not on gaming instructions, it shows that different aspects of video games greatly benefitted learners. Consequently, this framework posits that in-game instructions can bring impact to ESL vocabulary acquisition. The participants' reactions towards these in-game instructions are greatly enhanced by of motivation and curiosity. Therefore, there is a case for investigating the extent of ingame instructions in contributing to effective ESL vocabulary acquisition with motivation and curiosity as the intervening variables.

Building on the literature review and guided by this conceptual framework, this study seeks to answer the two research questions by specifically focussing on the direct relationship between in-game instructions and ESL vocabulary acquisition with motivation and curiosity as the contributing factors to vocabulary acquisition. 


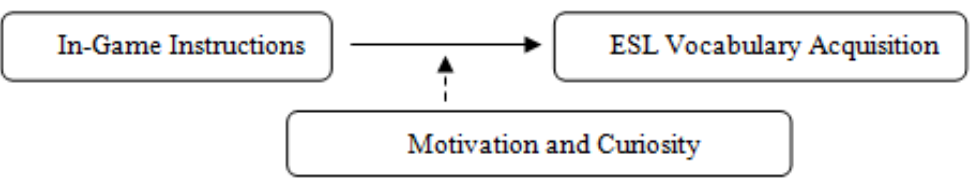

Fig. 6. Conceptual framework

\subsection{Research participants and location}

Ten participants from a sub-urban school in Pahang, Malaysia were selected for this study by using the purposive sampling method. These participants were selected based on their age, English examination results and responses to the verbal question related to their video game playing experience. These students were aged between 11 years and 12 years, had experience in playing video games and came from different ethnic groups, including Chinese and indigenous people. These participants also had different mother tongues of Malay or Chinese language but all had English as their second language.

A primary school in Maran, Pahang was selected as the study area. Pahang is the largest state in Peninsular Malaysia, and Maran is a district located at the state centre. This district is surrounded by isolated forests and oil palm plantations, whilst its residents came from different ethnic and religious groups.

\subsection{Research Instruments}

Observation checklist: A list containing eight questions was used to guide the researcher in the observations. The participants were referred to in the third person in these questions, which started with the words, 'Does he/she'. Three questions $(3,4$ and 7) pertained to the emotions of participants, whereas five questions $(1,2,5,6$ and 8) pertained to the actions taken by the participants. These questions explored the reactions of the participants towards the in-game instructions, especially in the aspects of motivation and curiosity. The speech, facial expressions and actions taken by the participants throughout their gameplay were all observed. Several yes or no questions were also formulated, and a blank column was placed beside these questions on which the researcher can write down her remarks.

The changes in the emotions (e.g. frustration or excitement) of the participants during their gameplay were recorded along with the actions they took to complete the game, their attitudes towards the words used in the game and the usefulness of ingame instructions.

Face-to-face interview: Six questions were used to measure the interest of the participants towards the game (Question 1), their perceptions towards the usefulness of in-game instructions in completing the in-game tasks (Question 2) and in enhancing vocabulary acquisition (Question 4), the difficulty of the game vocabulary for the participants (Question 3) and the motivation of the players in the game 
(Questions 5 and 6). Questions that elicited their feelings were initiated. This was followed by questions about their opinions towards the in-game instructions.

Pre- and post-game vocabulary tests: A vocabulary test including 40 words and definitions was built based on the game instructions. This test was created by the researcher after playing the game, taking notes, selecting the most frequently appearing words in the game and adding some distractions.

The above questions aimed to test the participants' understanding and retention of the meanings of words. The participants were asked to complete a matching type test where they had to match each listed word to each listed meaning. These words were randomly presented in the pre- and post-tests and were evaluated by using a few types of questions, including words translation and multiple-choice questions. Instead of word-for-word manual translation by participants, a list of meanings which were written in both Malay and Chinese language was provided for selection and matching purposes. Participants are therefore able to refer to any language that they are most comfortable with. With that, a total of 25 questions were formulated for the wordsmeanings matching, 10 questions were formulated for multiple-choice questions and 15 questions were formulated for fill-in-the-blank questions.

\subsection{Reliability and validity}

Reliability and validity are the two most important qualities of any research instrument. Reliability refers to the ability of a measure to remain constant over a certain period despite the presence of other potential influential factors [20]. A pilot test for the three instruments was conducted. The questions in the observation checklist and interviews were cross-checked and validated by three primary school teachers to strengthen the validity of these measurements to study the motivation and curiosity of children when playing video games. A game vocabulary test was also given and tested by eight pupils in the school. The feedback collected during the pilot test was used to enhance the suitability of the research instrument. For instance, Cronbach's Alpha was utilised to estimate the reliability of the game vocabulary test in the study. Table 1 showed the item-analysis output from SPSS for the pre and post vocabulary tests.

Table 1. Items analysis from SPSS output

\begin{tabular}{|c|c|c|c|}
\hline \multicolumn{4}{|c|}{ Scale Statistics } \\
\hline Mean & Variance & Std. Deviation & N of Items \\
\hline 35.3000 & 134.233 & 11.58591 & 2 \\
\hline
\end{tabular}

\begin{tabular}{|l|c|c|c|c|}
\hline \multicolumn{5}{|c|}{ Item-Total Statistics } \\
\hline & $\begin{array}{c}\text { Scale Mean if } \\
\text { Item Deleted }\end{array}$ & $\begin{array}{c}\text { Scale Variance if } \\
\text { Item Deleted }\end{array}$ & $\begin{array}{c}\text { Corrected } \\
\text { Item-Total } \\
\text { Correlation }\end{array}$ & $\begin{array}{c}\text { Squared } \\
\text { Multiple } \\
\text { Correlation }\end{array}$ \\
\hline Pre Vocabulary Test & 17.8000 & 45.733 & .812 & .660 \\
\hline Post Vocabulary Test & 17.5000 & 29.167 & .812 & .660 \\
\hline
\end{tabular}




\begin{tabular}{|c|c|c|}
\hline \multicolumn{3}{|c|}{ Reliability Statistics } \\
\hline Cronbach's Alpha & Cronbach's Alpha Based on Standardized Items & Nof Items \\
\hline .884 & .896 & 2 \\
\hline
\end{tabular}

Cronbach's alpha reliability coefficient normally ranges between o and 1 . The closer Cronbach's alpha coefficient is to 1.0, the greater the internal consistency of the items in the scale. Based on the result shown, the game vocabulary test was reliable with the alpha coefficient of reliability of .884 which according to George and Mallery [6] is considered as having good internal consistency. Three gameplay sessions were conducted within a month to ensure the reliability of the reactions and attitudes shown by the participants towards the in-game instructions. To increase the reliability of the game's vocabulary test, in Section A and C, the choices of the answer are randomly assigned. Hence, the guessing of the answers could be largely detected by observing the pre- and post-tests result for each question.

Validity refers to the extent that the research instrument assesses what it is meant to measure [5]. To ensure the validity of the research instruments, an observation checklist was designed by zooming into a few specific questions, which helped the researcher observe the reactions of the participants to the in-game instructions. The vocabulary test was also built based on the vocabulary of Criminal Case and not on the vocabulary of other video games, thereby providing a valid assessment of the vocabulary gained by the participants.

\subsection{Data collection}

The data were collected by using observation checklists, conducting face-to-face interviews and administering a game vocabulary test. By using the observation checklist, the three main aspects which were feelings, opinions towards in-game instructions and actions taken were recorded during the thirty minutes gameplay for each session. Three sessions were conducted within a one-month period. The researcher briefly introduced the Criminal Case game to the participants. Before and after playing this game for a month, the participants were asked to participate in a game vocabulary test, and the results of both tests were collected. A face-to-face interview was also conducted by using the questions provided. The complete procedure is illustrated in Figure 7.

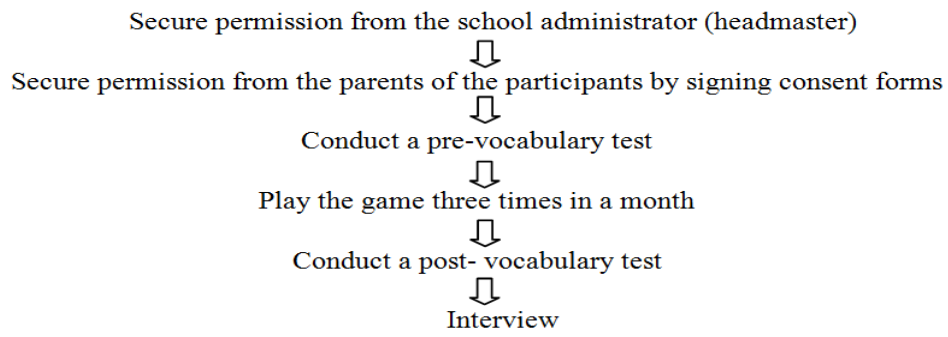

Fig. 7. Flowchart of the data collection procedure 


\section{Data Analysis and Results}

The pre- and post-test results were compared to determine the influence of playing video games on vocabulary acquisition. Table 2 shows the pre- and post- results for the participants.

Table 2. Pre-test and post-test results

\begin{tabular}{|l|c|c|c|c|c|}
\hline \multicolumn{1}{|c|}{ Participant* } & Pre-test & Percentage & Post-test & Percentage & Increment \\
\hline Loh & $22 / 50$ & $44 \%$ & $29 / 50$ & $58 \%$ & $+14 \%$ \\
\hline Ong & $12 / 50$ & $24 \%$ & $14 / 50$ & $28 \%$ & $+4 \%$ \\
\hline Hanum & $26 / 50$ & $52 \%$ & $31 / 50$ & $62 \%$ & $+10 \%$ \\
\hline Lim & $13 / 50$ & $26 \%$ & $11 / 50$ & $22 \%$ & $-4 \%$ \\
\hline Yong & $16 / 50$ & $32 \%$ & $18 / 50$ & $36 \%$ & $+4 \%$ \\
\hline Chang & $13 / 50$ & $26 \%$ & $14 / 50$ & $28 \%$ & $+2 \%$ \\
\hline Wong & $15 / 50$ & $30 \%$ & $15 / 50$ & $30 \%$ & $0 \%$ \\
\hline Sheng & $18 / 50$ & $36 \%$ & $20 / 50$ & $40 \%$ & $+4 \%$ \\
\hline Hern & $12 / 50$ & $24 \%$ & $8 / 50$ & $16 \%$ & $-8 \%$ \\
\hline Fiza & $20 / 50$ & $40 \%$ & $25 / 50$ & $50 \%$ & $+10 \%$ \\
\hline
\end{tabular}

*All participants' names are fictitious

As shown in Table 2, after playing the game for a month, seven participants showed some improvements in their recognition and understanding of game vocabulary. However, one participant showed a $2 \%$ decrement in the post test. These results indicate that the participants had about half or more than half of the unknown game vocabularies before the gameplay sessions are conducted.

Table 3. Observation and Interview Results

\begin{tabular}{|l|c|c|c|c|c|}
\hline \multicolumn{1}{|c|}{ Participant } & $\begin{array}{c}\text { Game } \\
\text { Engagement }\end{array}$ & $\begin{array}{c}\text { Try to change } \\
\text { language }\end{array}$ & $\begin{array}{c}\text { Read In-game } \\
\text { instructions }\end{array}$ & $\begin{array}{c}\text { Ask for } \\
\text { meanings }\end{array}$ & $\begin{array}{c}\text { Tell vocabulary } \\
\text { learnt }\end{array}$ \\
\hline Loh & YES & YES & YES & NO & NO \\
\hline Ong & NO & YES & NO & YES & YES \\
\hline Hanum & YES & YES & YES & NO & YES \\
\hline Lim & YES & YES & NO & NO & NO \\
\hline Yong & YES & NO & NO & NO & YES \\
\hline Chang & YES & YES & NO & NO & NO \\
\hline Wong & YES & YES & NO & YES & NO \\
\hline Sheng & YES & YES & YES & NO & YES \\
\hline Hern & NO & YES & NO & NO & NO \\
\hline Fiza & YES & NO & YES & YES & YES \\
\hline
\end{tabular}

Based on Table 3, the results of observation checklist were analysed based on participants' facial expression, body language, emotion, interest and attitudes throughout the gameplay. Upon its introduction, the game immediately piqued the interest of the participants. However, Loh, Hanum and Yong did not show great excitement while playing the game but rather remained as silent readers and gamers. They were focused on the game and seldom asked for the meanings of words they 
encounter. Meanwhile, Lim and Wong were very enthusiastic in playing the game. Their faces showed signs of nervousness and excitement as they were smiling and laughing throughout the game. Lim also showed other types of body language, including exercising his fingers and sitting properly whilst waiting for the game to start. Ong and Hern did not smile throughout the game and gradually became uninterested upon learning that the game language was English. Such behaviour can be clearly observed from their responses to questions, such as 'What is the meaning of this?', to which they often responded with a bored, frustrated tone. However, Ong gradually became interested in the game after quickly obtaining the needed items but Hern has a different reaction from her. She often said, 'Oh! I saw it before' and 'I know this'.

During the game, Ong and Lim requested to change the game language to Chinese as they could not understand the English instructions, which made them feel confused and frustrated. Furthermore, Loh and Hanum received stars for each scene just after two weeks of gameplay. In the interview, they admitted to have changed the game language from English to Chinese or Malay at home. Whilst the participants bent all the rules whenever they could, Yong and Fiza did not think about changing the game language. When asked whether they accept English as the game language, only Hanum, Loh and Fiza paused the game before viewing the next dialogue. These three were engrossed in completing their tasks, silently read the game instructions and continued with their tasks. From the interview results, they mentioned they scanned and chose to read some of the instructions in game, especially those with some known words. In this way, they emphasised that they could get a rough idea about the story in the criminal case.

All participants acquired limited to no vocabulary throughout their gameplay. Loh, Lim, Chang, Wong and Hern were unable to identify any word that they have learned from the game. Lim admitted that he felt discouraged, frustrated and disappointed due to his failure to recognise any of the words used in the game. Such feelings were also evident in his in-game activities; for instance, they took some time before answering any problems and kept avoiding eye contact with the interviewer. By contrast, Ong listed some of his learnt words (e.g. toilet paper, candy, backpack and postcards) but with some misspellings (e.g. cissors instead of scissors and 'what body' instead of dead body). Hanum learnt the word for can of paint, which she initially mistook for a pail, and learnt the correct spelling of the word 'scan', which she thought to be spelled as 'skan'. Yong remembered extra words from the game, including 'crow', 'target', 'tricycle', 'hanger', 'backpack' and 'electric razor'. Similarly, Sheng and Fiza learnt words such as 'guilty', 'fabric' and 'laboratory' although Sheng misspelled it as 'laboratery'.

\section{$5 \quad$ Discussion and Findings}

Can the in-game instructions in a non-educational game pique the curiosity of participants to acquire new words? 
In-game instructions take many forms to issue orders to the players. Therefore, this study only focuses on the interaction between the player and the pre-set game instructions. The findings show that only some in-game instructions were able to arouse the curiosity of the players.

\subsection{Omit Choices, On Demand}

Whenever they are given the chance, most of the players skip those lengthy and unnecessary instructions that do not contribute to their final game results. Instead, they only focus on those instructions that they need to understand to score or win the game. However, some players enjoy knowing as much detail about the game as possible similar to the two research participants who read most of the instructions presented in the game. In sum, the attitudes of players and their motives for playing a video game (e.g., 'for pure entertainment' or 'for learning') influence their vocabulary acquisition. Non-educational games can motivate players to learn if they give out information that can help them score and win the game and discourage them from ignoring the other instructions. For instance, such information must be provided at times when these players need to get clues or guidance to move to another level. In this study, to facilitate understanding, only some instructions were provided along with hints. Included are displaying pictures of objects mentioned or having the objects moved to assist players to find the items when participants clicked on the unknown words.

\subsection{Can in-game instructions familiarise participants with unknown words?}

Even though the findings of this study prove that in-game instructions in noneducational games can pique the curiosity of players to learn new words, these instructions are not helpful in familiarising these players with unknown words. In other words, these players had neither the 'breadth' nor 'depth' of vocabulary as mentioned by [33]. Language acquisition is considered successful when language knowledge is being transmitted in and out of the learners. The low interaction of players with the in-game instructions negatively affects their use and acquisition of new vocabulary. The participants merely 'see' and read these instructions without applying them, thereby leaving these instructions unclear to their minds. The postvocabulary test results prove that the research participants were unable to recognise some words used in the in-game instructions. Their reluctance in listing new words during the interview further supported this statement.

\section{Conclusion}

To a certain extent, the findings of this work indicate that the in-game instructions in non-educational games can motivate players to expand their vocabulary as the result showed that out of the ten participants, four read the in-game instructions and only three participants ventured to ask the definition of some words. Successful 
vocabulary acquisition triggered by in-game instructions also does not necessarily translate to higher motivation among players.

This research was largely based on qualitative research methods. The chosen research instruments did not, for the most part, yield statistically significant results. While the study was rigorously conducted and validated, the results are limited by the sample size. For future studies, a larger population would enable generalisation of result and allow substantial claims. However, this study serves as a stepping stone and provided a framework for research on a larger scale.

This study contributes to teaching pedagogy as well in the educational field by highlighting the usefulness of in-game instructions in the vocabulary acquisition of players. Future works may investigate the in-game instructions being used in other non-educational games to verify the usefulness of these texts in motivating vocabulary acquisition. A similar yet longer study must also be carried out to further understand the impact of in-game instructions on vocabulary retention. Different age groups and geographical locations may also be considered to generate novel insights.

\section{$7 \quad$ Acknowledgement}

The authors received financial support for the research and authorship of this article from the Malaysian Ministry of Education Research Grant coded FRGS/1/2018/SS09/UKM/02/1. Much thanks is also due to Ganter Management and Consultancy Sdn. Bhd. for their support in this research.

\section{$8 \quad$ References}

[1] American Psychiatric Association, Diagnostic and statistical manual of mental disorders. Fifth edition. Arlington, VA: Author, 2013.

[2] Ari-Pekka Vaisanen, "Learning English through Video Games: Finnish Learners' Experiences," M.S. thesis, University of Jyvaskyla, 2018.

[3] Bandura, "Growing Primacy of Human Agency in Adaptation and Change in the Electronic Era," European Psychologist, vol. 7, no.1, pp. 2-16, Hogrefe \& Huber Publishers, 2002. https://doi.org/10.1027//1016-9040.7.1.2

[4] C. Marshall, "Face-to-Face Interviews - Advantages and Disadvantages," 2016. [Online], Available:https://www.linkedin.com/pulse/face-to-face-interviews-advantagesdisadvantages-charlie-marshall. [Accessed March 28, 2019].

[5] C. Robson, Real World Research: A Resource for Users of Social Research Methods in Applied Settings. Second Edition, Sussex, A. John Wiley and Sons Ltd., 2011.

[6] D. George \& P. Mallery, SPSS for Windows step by step: A simple guide and reference. 11.0 update (4th ed.). Boston: Allyn \& Bacon, 2003. https://doi.org/10.4 $\underline{324 / 9781351033909}$

[7] E. Akman \& R. Cakir, "Pupils' Opinions on an Educational Virtual Reality Game in Terms of Flow Experience," International Journal of Emerging Technologies in Learning, vol.14, no.15, 2019, [Online], Available: https://www.online-journals.org/index.php/ijet/article/view/10576. [Accessed July 5, 2019]. https://doi.org/10.3991/ijet.v14i15.10576 
[8] H.B. Duran, "How Different Generations Play Video Games, From Platforms To Genres," A. List, 2017. [Online], Available: https://www.alistdaily.com/strategy/differentgenerations-play-video-games-platforms-genres/. [Accessed Feb 26, 2019].

[9] I. Akhtar, "Research Design," Research in Social Science: Interdisciplinary Perspectives, pp. 69-84, 2016.

[10] I. Granic, A. Lobel, \& C. M. E. Rutger, "The Benefits of Playing Video Games," American Psychologist, vol. 69, no. 1, pp. 66-78, 2014. https://doi.org/10.1037/a0034857

[11] J. DeHaan, W. M. Reed, \& K. Kuwada, "The effect of interactivity with a music video game on second language vocabulary recall," Language Learning and Technology, vol. 14, no. 2, pp. 74-94, 2010, [Online], Available: https://eric.ed.gov/?id=EJ895974. [Accessed March. 5, 2019].

[12] J.L. Plass, B.D. Homer, \& K. K. Charles, "Foundations of Game-Based Learning," Educational Psychologist, vol. 50, no. 4, pp. 258-283, 2015. https://doi.org/10.1080/0 $\underline{0461520.2015 .1122533}$

[13] J. P. Gee, What video games have to teach us about learning and literacy, New York (N.Y.): Palgrave Macmillan, 2003. https://doi.org/10.5040/9781628927924.ch-013

[14] J. P. Gee, \& E. R. Hayes, Language and learning in the digital age. London, UK: Routledge, 2011.

[15] J.P. Gee, Foreword. In Reinders, H. (Ed.), Digital games in language learning and teaching. pp. xii-xiv. London: Palgrave Macmillan, 2012.

[16] J. P. Gee, Good video games and good learning: Collected essays on video games, learning and literacy. Second edition. New York: Peter Lang, 2013. https://doi.org/10.19 173/irrodl.v8i3.498

[17] K. Dini, "On video games, culture, and therapy," Psychoanalytic Inquiry 32, pp. 496-505, 2012.

[18] L. Thorne Steven, Ingrid Fischer and Xiaofei Lu, "The semiotic ecology and linguistic complexity of an online game world," ReCALL, vol. 24, pp. 279-301, 2012. [Online], Available: https://www.cambridge.org/core/journals/recall/article/semiotic-ecology-andlinguistic-complexity-of-an-online-game-orld/895B505C7298D7E1A1B8734AA0E3C8E2 https://doi.org/10.1017/s0958344012000158

[19] M. Alqahtani, "The importance of vocabulary in language learning and how to be taught," International Journal of Teaching and Education III(3), pp. 21-34, 2016. https://doi.org/10.20472/te.2015.3.3.002

[20] M. J. Allen, \& W. M. Yen, Introduction to Measurement Theory. Monterey, CA: Brooks/Cole Publishing Company, 1979.

[21] R. Garris, R. Ahlers, J. E. Driskell, "Games, motivation, and learning: A research and practice model," In: Simulation and Gaming, vol. 33, no. 4, December 2002, pp. 441-467. https://doi.org/10.1177/1046878102238607

[22] S. Gerber, \& L. Scott, "Gamers and gaming context: Relationships to critical thinking," British Journal of Educational Technology, vol.42, no. 5, pp. 842-849. Blackwell Publishing, 2011. https://doi.org/10.1111/j.1467-8535.2010.01106.x

[23] S. L. Thorne, I. Fischer, \& X. Lu, "The semiotic ecology and linguistic complexity of an online game world," ReCALL, vol. 24, no. 3, pp. 279-301, 2012. https://doi.org/10.10 $17 / \mathrm{s} 0958344012000158$

[24] S. Schwan, "Game Based Learning," e-teaching.org, 2006. [Online], Available: https://www.e-teaching.org/didaktik/konzeption/meth oden/lernspiele/game_based_learning/gamebasedlearning.pdf. [Accessed April 16, 2019]. 
[25] Y.L. Tsai, \& C.C. Tsai, "Digital game-based second-language vocabulary learning and conditions of research designs: A meta-analysis study," Computers \& Education, vol. 125, pp. 345-357, 2018. https://doi.org/10.1016/j.compedu.2018.06.020

\section{Authors}

Tan Pei Qin is a master student of Faculty of Education in National University of Malaysia, Bangi, Selangor, Malaysia. She is teaching English as a Second language (ESL) at a primary school in Maran, Pahang, Malaysia.

Tan, Kim Hua (Ph.D), an Assoc Professor of Applied Linguistics at the Centre of Language Research and Linguistics, Faculty of Social Sciences and Humanities, Universiti Kebangsaan Malaysia. Her research interests are in digital humanities, including corpus-driven research, corpus lexicography and research looking at the interrelationship between language, power and ideology. Her most recent paper in 2019 'The "Geopolitical" Factor in the Syrian Civil War: A Corpus-Based Thematic Analysis' is published in Sage Open.

Article submitted 2019-09-09. Resubmitted 2019-11-18. Final acceptance 2019-11-18. Final version published as submitted by the authors. 